\title{
Originalarbeiten
}

\section{Zur Sterbehilfediskussion in der theologischen Ethik}

\author{
Markus Zimmermann-Acklin
}

\section{Reflections on Euthanasia in Theological Ethics}

\begin{abstract}
Definition of the problem: In parallel with public and philosophical discussions, there is an ongoing debate about euthanasia in theological ethics. In this situation it is helpful to provide a systematic overview of the different arguments and judgements. Arguments: A short introduction is followed by a first part dealing with the definition of terms. The second and major part concerning normative arguments is subdivided into three steps: the level of principles, the more concrete level of actions and finally the level of social consequences. The third part presents some reflections following the eudaimonistic ethical tradition (the ethics of a good life and death) focusing on the central question: how to find meaning in life when faced with suffering. Conclusion: It would be fruitful to intensify the ethical reflection both on the problems concerning the foundations of social life, especially the meaning of dignity, autonomy and inviolability of human life, and social consequences of a liberal practice of euthanasia. In this context ethicists are challenged to reflect on the different approaches to what is or can be a good life and a human death.
\end{abstract}

Key words: Euthanasia - Theological ethics - Normative arguments - Suffering

Zusammenfassung. Vergleichbar mit den öffentlichen und philosophischen Debatten wird die Sterbehilfethematik gegenwärtig auch in der theologischen Ethik kontrovers diskutiert. Im Anschluss an eine kurze Klärung der Begriffe geht es im Hauptteil dieses Beitrags um eine Darstellung und Beurteilung der wichtigsten normativen Denkfiguren aus theologisch-ethischer Sicht. Dabei werden Argumente auf der Prinzipienebene, der Handlungsebene und der Ebene sozialer Prozesse voneinander unterschieden. Mit Überlegungen in der strebensethischen Tradition oder der Ethik des guten Lebens wird in einem abschließenden Teil die Aufmerksamkeit auf die Sinnfrage angesichts des Leidens gerichtet. Die ethische Diskussion wird sich einerseits mit den Grundlagen unseres Zusammenlebens, insbesondere der Bedeutung von Würde, Autonomie und Unantast-

Markus Zimmermann-Acklin

Moraltheologisches Institut, Universität Fribourg, Rue St. Michel 6, CH-1700 Fribourg, Switzerland 
barkeit menschlichen Lebens und andererseits mit den Fragen möglicher sozialer Auswirkungen einer liberalen Sterbehilfepraxis beschäftigen müssen. Daneben besteht eine Herausforderung darin, die Pluralität der Vorstellungen von einem guten Leben und Sterben wahrzunehmen und die Verständigung darüber zu suchen.

Schlüsselwörter: Sterbehilfe - Euthanasie - Theologische Ethik - Normative Diskussion - Leiden

\section{Einführung}

Ängste vor chronischen oder unerträglichen Schmerzen am Lebensende, dem Verlust der Eigenständigkeit, der Selbstkontrolle oder des klaren Verstandes, schließlich Ängste vor einer Überbehandlung in der Terminalphase haben bewirkt, dass die Gestaltung der Sterbephase und insbesondere die verschiedenen Formen der Sterbehilfe gegenwärtig zu Themen kontrovers geführter Debatten geworden sind. Bedeutende Begleitumstände dieser Auseinandersetzungen bestehen in der zunehmenden Technisierung der Medizin, der Infragestellung der ärztlichen Autorität, dem ökonomischen Druck im Gesundheitswesen, dem Aufbau der Hospizbewegung und hier insbesondere der palliativen Betreuung, den weltweiten Aktivitäten der Sterbehilfegesellschaften, der Einführung von Patientenverfügungen und der in verschiedenen Ländern vorliegenden politischen Initiativen zur Veränderung des Strafrechts in Sachen aktiver Sterbehilfe.

Während Beiträge aus theologischer Perspektive die medizinethischen Diskussionen zu Beginn der Bioethik-Renaissance in den sechziger und siebziger Jahren noch weitgehend bestimmten, werden sie gegenwärtig mit Hinweis auf ihre Pluralismusuntauglichkeit nicht selten marginalisiert [15]. Innerhalb der theologischen Ethik bewirkt diese Entwicklung zwei entgegengesetzte Reaktionen: Einerseits wird die Verständigung mit verschiedenen Positionen und Weltanschauungen gesucht, indem ein Schwerpunkt auf die normativen Auseinandersetzungen gelegt und eigene Traditionen zugunsten eines „moral esperanto“ in den Hintergrund gestellt werden (universalistische Variante), andererseits werden im Zeichen des Kommunitarismus Neuansätze in der expliziten Betonung der religiösen und insbesondere konfessionellen Besonderheiten gesucht (partikularistische Variante) ${ }^{1}$. Parallel zu entsprechenden philosophischen Neuansätzen bestehen die Probleme der jeweiligen Zugänge bei universalistischen Theorien in einer Abspaltung von kontextuellen Elementen und bewährten Traditionen - letztlich einer „Spaltung des Ichs“ -, bei partikularistischen Ansätzen in einer Immunisierung oder Abschottung vor Kritik und einem Hang zum Fundamentalismus. Meines Erachtens schließen die beiden Denkbewegungen, wie sie auch in der Auseinandersetzung um die Sterbehilfe zum Ausdruck kommen, einander nicht aus, sondern sind komplementär zu verstehen und stellen Antwortversuche auf dasselbe gesellschaftliche Problem dar: die Suche nach

\footnotetext{
1 Vgl. beispielsweise die von Tristram Engelhardt Jr., Andrew Lustig und Kevin Wildes 1995 gegründete Zeitschrift «Christian Bioethics. Non-Ecumenical Studies in Medical Morality»: Den im Kontext der amerikanischen Bioethik bekannten Herausgebern geht es nicht nur um die Profilierung einer religiösen Ethik, sondern darüber hinaus auch um die Betonung der Unterschiede zwischen verschiedenen konfessionellen Zugängen zu medizinethischen Fragen [9]
} 
ethisch vertretbaren Entscheidungen in multikulturell zusammengesetzten, pluralistischen Gesellschaften. ${ }^{2}$

Im Anschluss an eine Klärung der zentralen Begriffe geht es mir im zentralen Teil dieses Beitrags um eine Beurteilung der normativen Argumentationsfiguren, die in der gegenwärtigen Sterbehilfedebatte eine wichtige Rolle spielen. Dies geschieht aus der Perspektive der katholischen Moraltheologie, welche im Rahmen naturrechtlicher Ansätze traditionell eher die universalistische Variante bevorzugt hat und davon ausgeht, dass die Theologie im Bereich der normativen Auseinandersetzungen über keinerlei Sondergut verfügt. Daraus folgt jedoch nicht, dass sie sich im Rahmen dieser normativen Debatten nicht zu beteiligen hätte: Bereits die Entdeckung normativ relevanter Fragen, die nötige Sensibilität für die ,Zeichen der Zeit“" und auch Fragen der Motivation in der Umsetzung der gefundenen Einsichten (die Moralpragmatik) gehören zu den wichtigen Aufgaben einer aus theologischer Perspektive betriebenen biomedizinischen Ethik. In einem abschließenden Teil werde ich kurz die Frage aufgreifen, die angesichts der Gestaltung der Sterbephase in strebensethischer Tradition auftaucht, nämlich die Frage nach dem Sinn des Lebens angesichts aussichtslosen Leidens. In diesem Bereich der Fragen nach den Vorstellungen von einem guten Leben und damit auch von einem guten Sterben gewinnen mit den Sinnfragen auch religiöse Aspekte und damit partikulare Traditionen an Bedeutung.

\section{Zum Sterbehilfebegriff}

Unter Sterbehilfe verstehe ich eine Tötung oder ein Sterbenlassen eines schwer leidenden oder sterbenden Menschen auf dessen Verlangen oder zu dessen Wohl. Damit werden zunächst eugenisch begründete Tötungsaktionen im Sinne der Nationalsozialisten vom Begriff ausgeschlossen, die als „unfreiwillige Tötung“ bzw. korrekterweise als Mord zu bezeichnen sind. Was den Sterbenden angeht, bleibt unbestimmt, in welchem Krankheitsstadium er sich befindet und ob er zum Zeitpunkt seines Sterbens noch nicht oder nicht mehr entscheidungsfähig ist. Hinsichtlich der eingreifenden Person läßt die Definition Handlungsmotivation wie -intention weitgehend offen, ebenfalls, ob es um eine Tötung oder einen Behandlungsabbruch bzw. -verzicht geht, schließlich, ob diese Person ein Arzt sein muß oder beispielsweise auch ein Freund oder Verwandter sein kann.

Diese weite Begriffsbestimmung indiziert bereits die zu diskutierenden Probleme. Wird unter Sterbehilfe hingegen in sehr eingeschränktem Sinne, wie es beispielsweise in den Niederlanden geschieht, ausschließlich die ärztliche Tötung eines um seine Tötung bittenden Patienten verstanden, so benötigt man auf die Dauer - das zeigt der Verlauf der Diskussion auf deutliche Weise - einen Ersatz für das, was hier unter dem weiten Sterbehilfebegriff verstanden wird. Der niederländische Vorschlag, an dieser Stelle von „Medical Decisions Concerning the End of Life“ bzw. „Medical Practices Involving the End of Life“ (medizinische Entscheidungen oder Praktiken, die das Lebensende betreffen) zu sprechen, scheint weder praktikabel noch sehr präzise zu sein ([23],[24],[30]).

\footnotetext{
2 Vgl. dazu u.a. aus orthodoxer Sicht die Themenhefte [8] und [10], aus deutschsprachiger protestantischer Sicht außerdem ([7, 13, 11], S 63-94) und aus katholischer Sicht ([14, 16, 25, 28)
} 
Auch wenn es in den deutschsprachigen Ländern kaum möglich und derzeit nicht sinnvoll ist, den schillernden Sterbehilfebegriff durch den Euthanasiebegriff zu ersetzen, sprechen auf die Dauer zwei Gründe für eine Umorientierung, erstens ein historischer: Der Sterbehilfebegriff wurde erst zu Beginn dieses Jahrhunderts als Äquivalent für die Tötung auf Verlangen geprägt, Fritz Mauthner kommentierte den Neologismus damals lakonisch als „,Vergeudung der Sprachenergie", sprachgeschichtlich hat er offensichtlich nichts mit der medizinischen oder seelsorgerlichen Sterbebegleitung zu tun. Zweitens ein praktischer: In vielen Sprachen besteht zudem keine sinnvolle Parallele zum deutschen Sterbehilfebegriff, so dass man in der internationalen Diskussion - beispielsweise in den lehramtlichen Äußerungen der katholischen Kirche [18] oder im mehrsprachigen schweizerischen Kontext - stets auf den Euthanasiebegriff angewiesen ist. Um Missverständnisse zu vermeiden, gebrauche ich deshalb beide Begriffe gleichbedeutend. Auf die eigens zu diskutierende Abgrenzung der ärztlichen Suizidbeihilfe von der Euthanasie möchte ich nicht weiter eingehen. Es spricht allerdings einiges dafür, dass eine eindeutige Grenzziehung kaum möglich ist und daher die weiteren Überlegungen durchaus auf beide Handlungsbereiche bezogen werden können [29].

\section{Hauptargumente in der normativen Diskussion}

Zugunsten einer systematischen Übersicht lassen sich die wichtigen Argumentationsfiguren, die in der Sterbehilfedebatte auftauchen, in drei Kategorien einteilen, nämlich in Argumente auf der Prinzipienebene, der Handlungsebene und der Ebene sozialer Prozesse [28]. Diese Aufteilung entspricht einem Gang von den abstrakten oder empirisch neutralen zu den konkreten, weitgehend empirisch bestimmten Denkfiguren. Genuin theologische Überlegungen werden insbesondere auf der Prinzipienebene formuliert, insofern hier schöpfungstheologische Argumente oder auch - auf dem Hintergrund theonomer ${ }^{3}$ Ansätze - lehramtlich-autoritative Argumente vertreten werden.

\subsection{Prinzipienebene}

Zunächst ist hier die Berufung auf die Autonomie des Patienten zu erwähnen, wobei Autonomie im Sinne eines grundlegenden Rechts auf Selbstbestimmung verstanden wird: Dieses Recht wird insofern geltend gemacht, als ein Mensch in einer Grenzsituation die Entscheidung für sich treffen kann, nicht mehr weiterleben zu wollen; eine Entscheidung also, von deren Folgen in erster Linie er selbst betroffen ist.

Dagegen haben sich in den letzten Jahren Stimmen erhoben, welche auf die Grenzen menschenmöglicher Autonomie verweisen und das individualistische Menschenbild in Frage stellen, welches hier zugrunde liegt. Dabei wird auf die

\footnotetext{
${ }^{3}$ Theonomie (wörtlich: von Gott gegebene moralische Gesetze) setzt in diesem Kontext im Unterschied zur Autonomie (aus der Vernunft herzuleitende moralische Gesetze) den Akzent auf Gottes Autorität bzw. die Fehlbarkeit und Grenzen menschlicher Vernunfterkenntnis. Der entsprechende Begriff einer ,autonomen Moral“, der in der katholischen Moraltheologie der letzten dreißig Jahre eine bedeutende Rolle gespielt hat, ist nicht mit dem medizinethisch geläufigen, im Sinne der Selbstbestimmung verstandenen Autonomiebegriff zu verwechseln
} 
Kontextgebundenheit jeder Entscheidung verwiesen und das Fürsorge- und Nicht-Schadensprinzip dem Autonomieprinzip zur Seite gestellt. In diesem Zusammenhang ist an die Beiträge der feministischen Bioethik zu denken, die analog zu anderen kontextuellen, z.B. kommunitaristischen oder narrativen Ansätzen in der biomedizinischen Ethik - derzeit in der englischsprachigen Welt diskutiert werden ([16, 27]). Es wird überlegt, inwiefern Frauen anders als Männer von der Praxis der Sterbehilfe betroffen sind. Beobachtungen wie die, dass die meisten berühmten Fallbeispiele in der Literatur Frauenbeispiele sind bzw. drei Viertel der von Jack Kevorkian in den Tod begleiteten Menschen Frauen waren, geben Anlass zu fragen, ob die Arzt-Patienten-Beziehung in der Sterbehilfesituation von den üblichen Charakteristika der Mann-Frau-Beziehung geprägt ist. Insofern Frauen zudem finanziell häufig benachteiligt sind, weitaus öfter an Depressionen leiden als Männer und diejenigen sind, welche ihre Männer im Sterben begleiten, selbst aber nicht selten ohne familiäre Hilfe dastehen, zählen sie weitaus häufiger zu der Gruppe von Menschen, die um eine Suizidbeihilfe oder eine Tötung bitten könnten. Hinsichtlich der prinzipiellen Überlegung wird daraus die Befürchtung abgeleitet, dass es die Schwächeren in der Gesellschaft sein werden, welche durch die einseitige Betonung der Autonomie benachteiligt werden.

Ein zweites Argument lautet: Das ärztliche Töten verletze die Unantastbarkeit oder Heiligkeit menschlichen Lebens. Letztlich beruft man sich hier auf eine grundlegende Intuition, eine tiefsitzende moralische Überzeugung, die nicht unabhängig von anthropologischen oder theologischen Annahmen begründet werden kann. Dass diese Denkfigur nicht nur in theologischen, sondern durchaus auch in säkularen Ansätzen eine bedeutende Rolle spielt, hat zuletzt Ronald Dworkin gezeigt [6]. Sie beruht im wesentlichen auf einem Menschenbild, in welchem der Lebensschutz in jeder Hinsicht und unabhängig von bestimmten Qualitätsstandards eine wichtige Rolle spielt bzw. der Leib-Seele-Dualismus zugunsten eines ganzheitlichen Verständnisses stark relativiert wird.

In diesem Argumentationszusammenhang sind auch einige schöpfungstheologische Aussagen von Bedeutung. Diese lauten etwa: der Mensch übernehme die Rolle Gottes, wenn er töte (Missachtung der Souveränität Gottes als Herr über Leben und Tod), er missachte das Leben als Geschenk Gottes oder - hier vielleicht am naheliegendsten - er verkenne seine Befindlichkeit als gottebenbildliches Geschöpf, als „Imago Dei“. Letztlich handelt es sich hier also um theologische Sätze, Aussagen auf der Ebene von Gottes- und Menschenbildern und damit auf einer existentiellen Ebene, die nicht mit normativen Aussagen gleichgesetzt werden können. Wenn geschrieben steht, Gott entscheide über Leben und Tod, nicht der Mensch (Deuteronomium 32, 39; parallel: Sure 2, 259 im Koran), so ist dies eine existentielle Aussage auf der Ebene von Lebensentwürfen, nicht aber eine normative Anweisung für eine Dilemmaentscheidung am Krankenbett $([5,21])$. Ein Gegenentwurf zu einer Ethik, die im Sinne einer persönlichen Bezogenheit des Menschen auf Gott verstanden wird („Gott allein entscheidet über Leben und Tod"), lautet also nicht, der Mensch entscheide autonom und vernunftgemäß. Auf die vernünftige und damit autonome Entscheidung der Handelnden bleibt jede kognitive Ethik letztlich angewiesen. Ein Gegenkonzept müsste ebenfalls auf der existentiellen Ebene gesucht werden, liegt beispielsweise in Friedrich Nietzsches Weltbild des „Tollen Menschen“ aus der „Fröhlichen Wissenschaft“ vor, das folgendermaßen beschrieben wird: „Wer gab uns den Schwamm, um den ganzen Horizont wegzuwischen? Was taten wir, 
als wir diese Erde von der Sonne losketteten? Wohin bewegt sie sich nun? Wohin bewegen wir uns? Fort von allen Sonnen? Stürzen wir nicht fortwährend? Und rückwärts, seitwärts, vorwärts, nach allen Seiten? Gibt es noch ein Oben und ein Unten? Irren wir nicht wie durch ein unendliches Nichts? (...)“ ([20], S. 140)

Diese nihilistische Vision von Mensch und Kosmos ist im Kern ein Gegenbild zum biblischen Selbstverständnis des Menschen in seiner Bezogen- und Verwiesenheit auf einen personalen Gott. Aus beiden Weltanschauungen folgen nicht unmittelbar Normen für Dilemmaentscheidungen, beide schildern jedoch einen völlig anderen Bezugs- und Sinnrahmen, der sich letztlich auch auf wichtige Entscheidungen auswirken wird.

Entgegen dem Hinweis auf die Heiligkeit menschlichen Lebens wird meist anhand extremer Fallbeispiele für eine Lockerung des ärztlichen Tötungsverbots plädiert. Einem rigoristischen Verständnis der Unantastbarkeit werden die Lebensqualität und die Unterscheidung zwischen sinnvollem und sinnlosem Leben gegenübergestellt. Wie bereits bei der Abtreibungsdiskussion in den siebziger Jahren stehen hier die Schilderung besonders berührender Einzelschicksale von Patienten im Zentrum, die unter unerträglichen Schmerzen leiden, ohne jede Aussicht auf Besserung leben müssen, frei von depressiven oder anderen psychischen Verstimmungen sind und ihre Entscheidungsfähigkeit bewahrt haben. In dieser Perspektive ergeben sich mindestens zwei schwerwiegende Probleme: Erstens hat jede von außen vorgenommene Einteilung in sinnvolles und sinnloses Leben eine Diskriminierung bestimmter Gesellschaftsgruppen zur Folge und zweitens werden mit den geschilderten extremen Einzelschicksalen viele Menschen nicht repräsentiert, die in einer tatsächlichen Praxis der aktiven Sterbehilfe - wie der aktuellen niederländischen - betroffen sind, namentlich psychisch Leidende, Neugeborene mit schweren Schädigungen, Menschen außerhalb der Terminalphase, Demente oder Sterbende, die unter starken Depressionen leiden.

Ein drittes prinzipielles Argument besteht im Hinweis auf die zu wahrende Menschenwürde. Dieser Appell wird sowohl von Befürwortern wie Gegnern einer Relativierung des ärztlichen Tötungsverbots formuliert, zum einen verstanden als ein Anspruchsrecht (,Recht auf ein menschenwürdiges Sterben“ [14]), zum anderen als ein Schutzrecht (,Recht auf die Wahrung der Würde im Sterben“" [13]). Diese zunächst verwirrende Tatsache ist darauf zurückzuführen, dass im Begriff der Menschenwürde zwei unterschiedliche Traditionen zusammenkommen, welche einander in gewisser Hinsicht widersprechen: Einerseits wird die Würde in der Tradition Kants als eine anthropologische Grunddimension verstanden, welche unverlierbar ist und zur Begründung eines sehr weitreichenden Schutzes menschlichen Lebens führt („,Die Würde des Menschen ist unantastbar"); andererseits wird mit demselben Begriff eine Qualität assoziiert, welche dem Menschen als quasi äußere Gegebenheit zukommt und entsprechend auch verloren gehen kann (Würde im Sinne von Persönlichkeit, Charakter, Schönheit, Integrität etc.). ${ }^{4}$ Der Disput verweist letztlich auf die jeweils zugrundeliegenden Menschenbilder, die schon bei David Hume und den Stoikern einerseits, bei Immanuel Kant und vielen jüdischen, christlichen und muslimischen Denkern andererseits auszumachen sind. Die Grenzen bei der Verfügung

\footnotetext{
${ }^{4}$ Walter Lesch unterscheidet zwischen Tabuisierungs- und Statusfunktion des Würdebegriffs und erläutert die weitere Eingebundenheit dieser unterschiedlichen Würdeverständnisse und -funktionen in ihrem geschichtlichen und theoretischen Horizont ([19], S 32 - 37)
} 
über das menschliche Leben werden mit Verweis auf unterschiedliche Menschen- Welt- und Gottesbilder verschieden gesetzt und bleiben in der normativen Diskussion relativ unvermittelt nebeneinander stehen.

Schließlich bleibt viertens auf das Ärztebild hinzuweisen: Während einerseits ein Arzt- und auch Pflegebild entworfen wird, in welchem der Arzt und Pfleger als Auftragsempfänger verstanden werden, die für ihre Dienstleistung bezahlt werden und entsprechend dem Ethos bzw. Werteverständnis des Patienten handeln sollen, wird andererseits von einem stark von eigenen Werten geprägten Ärzteethos ausgegangen. Hier ist danach zu fragen, ob ein Ärztebild, welches die asymmetrische Situation in der Arzt-Patienten-Beziehung völlig außer Acht lässt, letztlich genügen kann. Es ist stark anzunehmen, dass die $\mathrm{Zu}$ weisung der ärztlichen Rolle in die eines Auftragempfängers das Engagement eines Arztes bei wichtigen Entscheidungen seines Patienten massiv unterschätzt.

\subsection{Handlungsebene}

De facto sind in der ärztlichen Praxis heute Entscheidungen zum Behandlungsverzicht oder -abbruch alltäglich geworden, während die Tötung auf Verlangen ein weithin anerkanntes Tabu darstellt. Wie ist diese unterschiedliche Beurteilung zu begründen? Befürworter der aktiven Euthanasie bezweifeln die Lauterkeit und Relevanz dieser unterschiedlichen Beurteilung von Töten und Sterbenlassen. In beiden Entscheidungssituationen seien vielmehr die ärztlichen Motive, Absichten und auch die Handlungsfolgen kaum oder überhaupt nicht voneinander zu unterscheiden: Mitleid, Linderung von unerträglichen Schmerzen, ärztliche Unterstützung in schweren Notsituationen, die angemessene Berücksichtigung der Patientenautonomie, der Tod des Betroffenen als erwünschte Folge spielten im Idealfall in beiden Fällen die entscheidende Rolle.

Rein deskriptiv unterscheiden sich beide Handlungen hinsichtlich des kausalen Anteils, den eine Ärztin am Tod eines Patienten hat: Beim Sterbenlassen greift eine Ärztin in den zum Tod führenden Prozess nicht ein, obgleich sie es könnte, beim Töten bringt sie den zum Tod führenden Prozess in Gang. Die sich hier anschließende schwierige Frage lautet: Ist dieser Unterschied moralisch dermaßen relevant, dass damit eine regelmäßige Praxis des einen akzeptiert und sogar vermehrt gefordert und ein totales Verbot des anderen begründet werden kann?

In der Begründung der moralischen Signifikanz der Unterscheidung spielen zunächst die erwähnten prinzipiellen Argumente eine entscheidende Rolle. So gibt es vier intrinsische Begründungsversuche, die alle Gegenargumenten ausgesetzt sind: Der erste Versuch behauptet die moralische Schlechtheit jeder Tötung. Töten sei eine in sich schlechte Handlung. In der Tradition wurden allerdings stets Ausnahmen von diesem intrinsece malum gemacht, ob in der Kriegssituation, bei der Selbstverteidigung oder, besonders widersprüchlich, bei der Todesstrafe. Der zweite beruft sich auf unsere Intuition: Intuitiv sei allen klar, dass die ärztliche Tötung in der Regel eindeutig verwerflicher sei als das Sterbenlassen, welches als Eingeständnis in die Grenzen der ärztlichen Kunst verstanden wird. Mit dem Hinweis auf die Intuition, so kritisieren die Gegner jedoch zurecht, wurde auch schon die Einführung des Frauenstimmrechts über Jahrhunderte erfolgreich verhindert oder stoßen heute homophile Paare auf gesellschaftlichen Widerstand. Ein dritter sucht den Umweg über das ärztliche 
Ethos: Eine tugendhafte Ärztin orientiere sich am hippokratischen Eid, sie könne daher das Töten von Patienten unmöglich in ihr ärztliches Selbstverständnis integrieren. Auch dieser Hinweis vermag jedoch nicht zu begründen, warum eine Tötung prinzipiell (d.h. immer und unter allen Umständen) schlechter sein soll als ein Sterbenlassen. Schließlich wird viertens auf die unterschiedliche Sinnstruktur von Töten und Sterbenlassen hingewiesen, und zwar mit der Behauptung: Die Sinnrichtung der Tötung stehe stets im Widerspruch zu der vermeintlich humanitären Motivation. Mitleid und Tötung ließen sich nicht miteinander vereinbaren, der Arzt werde sonst unberechtigterweise zum Richter über Leben und Tod [12]. Hier bleibt zu fragen, ob er das nicht ähnlich auch bei einer Entscheidung zum Behandlungsabbruch wird bzw. welcher Stellenwert in diesem Konzept dem Wunsch des Betroffenen eingeräumt wird.

Daneben werden extrinsische Begründungen für eine unterschiedliche Bewertung genannt, d.h. mit der Unterscheidung zwischen Töten und Sterbenlassen kovariierende Faktoren. Diese beziehen sich auf Argumente auf der Ebene sozialer Prozesse und bleiben damit stets vorläufig und revidierbar; sie betreffen nicht die Unterscheidung schlechthin. Meines Erachtens sind sie jedoch in verschiedener Hinsicht plausibel und haben sich nicht zufällig in der erwähnten intuitiv begründeten Ablehnung der Tötung Sterbender bei vielen Menschen verfestigt, allen voran bei den praktizierenden Ärztinnen und Ärzten. Es ist nämlich nicht von der Hand zu weisen, dass die Einführung der ärztlichen Tötung auf Verlangen als gesellschaftlich anerkannte Praxis eine Reihe unerwünschter Folgen nach sich ziehen könnte, welche die Praxis des Sterbenlassens nicht ohne weiteres hervorruft. $\mathrm{Zu}$ nennen sind beispielsweise: Eine negative Entwicklung des ärztlichen Selbstverständnisses, eine Störung des Vertrauensverhältnisses zwischen Arzt und Patient und schließlich mögliche Missbrauchs- und Ausweitungsgefahren. Angesichts dieser drohenden Konsequenzen, die im nächsten Punkt (3.3) noch näher erläutert werden, kann der Berufung auf die moralische Äquivalenz von ärztlichem Töten und Sterbenlassen nur mit großer Skepsis begegnet werden ([2], S 348-373; [22].

Neben diesem kausaltheoretischen Zugang („Causing the Death Account") besteht ein anderer Weg darin, die Sterbehilfeproblematik über die Intention des Arztes (,Intending the Death Account") anzugehen [3]. Die nicht selten missverstandene katholische Lehrtradition kann als Beispiel herangezogen werden, insofern hier bei der Beurteilung der Euthanasie neben der Berücksichtigung naturrechtlicher Aspekte in erster Linie der handelnde Arzt mit seinen Intentionen in den Blick genommen wird [18]. Dieser intentionstheoretische Zugang ist nur im Zusammenhang mit dem Prinzip der Handlung mit Doppelwirkung zu verstehen, welches im Verständnis der katholischen Moraltheologie das Tötungsverbot als Voraussetzung annimmt. Unter Zuhilfenahme dieses Doppelwirkungsprinzips wird bei Handlungen, die gleichzeitig erwünschte und unerwünschte Folgen nach sich ziehen, zwischen beabsichtigten und blo $\beta$ zugelassenen Konsequenzen unterschieden. Im ersten Fall spricht man von direkter, im zweiten von indirekter Sterbehilfe. So kann es passieren, dass in Folge einer gebotenen Schmerz- oder Symptomtherapie der Arzt Einfluss auf den Todeszeitpunkt seines Patienten nimmt, ohne dies zu beabsichtigen. Besonders umstritten diskutiert werden in der gegenwärtigen Auseinandersetzung die Fragen, wie ein sinnvoller Intentionsbegriff zu definieren sei und inwiefern die Berufung auf die subjektive Absicht eines handelnden Arztes als Grundlage für eine objektive Verbotsnorm dienen kann, welche lautet: Absichtliche Tötungen sollen immer 
verboten, bloß zugelassene Tötungen als Nebenwirkungen einer Schmerz- oder Symptomtherapie dagegen erlaubt sein.

Im Rahmen der medizinethischen Auseinandersetzungen ist von Bedeutung, dass die direkte und indirekte Euthanasie keine Sonderfälle der aktiven und passiven Sterbehilfe sind, wie meist behauptet wird, sondern eine andere Handlungstheorie voraussetzen und somit einen eigenen Zugang zum gesamten Problemfeld bieten. Schematisch lässt sich das folgendermaßen aufzeigen:

\begin{tabular}{|l|l|l|}
\hline & $\begin{array}{l}\text { Töten } \\
\text { (aktiv) }\end{array}$ & \multicolumn{1}{|c|}{$\begin{array}{l}\text { Sterbenlassen } \\
\text { (passiv) }\end{array}$} \\
\hline $\begin{array}{l}\text { Beabsichtigen } \\
\text { (direkt) }\end{array}$ & $\begin{array}{l}\text { [1] Eine Tötung in der klaren Absicht, } \\
\text { das Leben eines Menschen zu beenden. }\end{array}$ & $\begin{array}{l}\text { [3] Das Abbrechen } \\
\text { oder Unterlassen } \\
\text { einer Behandlung } \\
\text { in der klaren Absicht, } \\
\text { den Menschen sterben } \\
\text { zu lassen }\end{array}$ \\
\hline $\begin{array}{l}\text { Bloß zulassen } \\
\text { (indirekt) }\end{array}$ & $\begin{array}{l}\text { [2] Die nicht beabsichtigte Tötung } \\
\text { eines Menschen, die als bloße Nebenwirkung } \\
\text { einer Behandlung in Kauf genommen wird. }\end{array}$ & $\begin{array}{l}\text { [4] Das Abbrechen } \\
\text { oder Unterlassen } \\
\text { einer Behandlung } \\
\text { unter Inkaufnahme eines } \\
\text { nicht beabsichtigten, } \\
\text { früher einsetzenden } \\
\text { Sterbeprozesses }\end{array}$ \\
\hline
\end{tabular}

Abb. 1. Schema zum Verhältnis der Aktiv-Passiv- zur Direkt-Indirekt-Unterscheidung

In der ersten Zeile ist die Aktiv-Passiv-Unterscheidung eingetragen: Hier geht es um die Handlung, den Akt als solchen, unabhängig von subjektiven Anteilen des Handelnden. In der ersten Spalte dagegen ist die Direkt-Indirekt-Unterscheidung eingetragen, bei welcher es mit Betonung der Absicht des Handelnden weniger um die Handlung selbst als um die Intentionen des Arztes geht. Wird nun die Aktiv-Passiv-Unterscheidung als Zugang zum Thema gewählt - die passive Sterbehilfe als moralisch erlaubt, die aktive als verboten beurteilt - so gerät man angesichts der indirekten aktiven Sterbehilfe in Argumentationsnot, insofern diese nicht selten sogar zur ärztlichen Pflicht zu zählen ist. Wählt man hingegen die Direkt-Indirekt-Unterscheidung als Zugang - verbietet alle direkten und erlaubt alle indirekten Formen der Sterbehilfe - so bleibt man Erklärungen dafür schuldig, dass zur Bestimmung der moralischen Richtigkeit der passiven Sterbehilfe die Intention völlig bedeutungslos ist bzw. beim Zugang über die Handlungsintention die Patientenautonomie unberücksichtigt bleibt.

Meines Erachtens ergänzen sich beide Modelle, haben beide Vor- und Nachteile, und stellen deshalb ein Ethikkonzept in Frage, welches losgelöst vom Handelnden allein aufgrund von Handlungsbeschreibungen und den Folgen zu einem Urteil über die moralische Richtigkeit oder Falschheit eines Aktes gelangen will ([28], Kap. 5). Obgleich auf dieser Ebene der Einzelhandlungen in der Diskussion viel Energie aufgewendet wird, scheinen die wichtigen moralischen Entscheidungen nicht hier, sondern auf der Prinzipienebene und der Ebene sozialer Prozesse zu fallen. Das relativiert zumindest die nicht immer ganz einfach nachzuvollziehenden und zum Teil hitzig geführten Debatten um handlungstheoretische Aspekte der Sterbehilfediskussion. 


\subsection{Ebene sozialer Prozesse}

In der Auseinandersetzung um mögliche Auswirkungen auf gesellschaftlicher Ebene steht die Einführung einer ärztlichen Praxis der aktiven Sterbehilfe im Zentrum der Aufmerksamkeit. Die Grundfrage besteht darin, auf welcher Seite die Beweislast der Bedenklichkeit oder Unbedenklichkeit einer neu einzuführenden Praxis liegt, bei den Befürwortern oder Gegnern einer neuen Praxis. Hier besteht natürlich das zentrale Problem darin, wie etwas bewiesen werden kann, was in einem gesellschaftlichen Experiment ausprobiert werden müsste, bevor empirisch abgesicherte Aussagen möglich sind. In dieser Hinsicht erlauben erste Erfahrungen mit der Tötung auf Verlangen bzw. der ärztlichen Suizidbeihilfe in Ländern wie den Niederlanden, Oregon oder Australien erste Hinweise.

Im Rahmen der Auseinandersetzungen stehen zunächst die Bedeutung der unerträglichen Schmerzen und die Reichweite der Palliativmedizin zur Diskussion. Wird einerseits das Mitleid mit einem unerträglich leidenden Patienten als Hauptmotiv für eine Tötung auf Verlangen angegeben, wird andererseits behauptet, genau in dieser Situation verspreche die Palliativmedizin bzw. eine umfassende palliative Versorgung im Sinne der Hospizbewegung eine Alternative zur Tötung auf Verlangen, insofern Schmerzen gelindert und seelische Not aufgefangen werde [1]. Empirische Studien aus den Niederlanden, Oregon und Northern Territory geben hier ein erstes und deutliches Signal: Es zeigt sich, dass die zu ertragenden Schmerzen bei den Patienten, die um ihre Tötung bitten, de facto nicht zu den wichtigsten Motiven oder Beweggründen zählen. Vielmehr werden die Angst vor Verlust der Selbständigkeit, der Abhängigkeit von anderen und depressive Verstimmungen als Beweggründe genannt, auch die Angst vor unerträglichen Schmerzen ([4, 17, 23, 24, 26]). Offensichtlich geht es den betroffenen Patienten weniger um die Vermeidung unerträglicher Schmerzen als vielmehr um das Verhindern einer Lebensphase der Abhängigkeit von der Hilfe anderer Menschen.

Eine weitere Überlegung betrifft den möglichen Missbrauch, der im Falle einer ärztlichen Tötung fatale Konsequenzen nach sich zieht und kaum vollständig durch ein offizielles Kontrollsystem verhindert werden kann. Hier wird mit einigem Recht eingewandt, dass die Missbrauchsgefahr bereits bei der passiven Sterbehilfe immens hoch einzustufen sei und dort bereits heute weitreichende Folgen nach sich ziehe. In der Tat besteht in diesem Bereich des Behandlungsabbruchs und -verzichts heute ein großes Regelungsdefizit.

Eine bedeutende Debatte ringt sich zudem um die Überzeugungskraft verschiedener Slippery Slope-Argumente: Inwieweit lassen sich unerwünschte Ausweitungseffekte einer ärztlichen Tötungspraxis auf zunächst nicht anvisierte Bevölkerungsgruppen verhindern? Da solche Argumente einer unkontrollierbaren Ausweitung bei nahezu allen politischen Veränderungen auftauchen, stehen sie im Verdacht, Vorboten eines verknöcherten Konservativismus zu sein und werden deshalb von einigen Reform-Befürwortern völlig verworfen [14]. Im anderen Extremfall wird mit Verweis auf Dammbruchargumente, ganz besonders natürlich auf die Nazi-Verbrechen und deren mögliche Wiederholung, ein genereller Gesprächsabbruch verlangt und betrieben. Ich halte beide Strategien für unplausibel. Slippery Slope-Argumente funktionieren im ethischen Diskurs als Elemente politisch-gesellschaftlicher Klugheit: je überzeugender sie begründet werden, desto eher sollten sie ernst genommen werden und können die Beweislast der Unbedenklichkeit einer einzuführenden Praxis auf die Seite der Befür- 
worter abwälzen. Angesichts der Erfahrungen in den angesprochenen Ländern bestehen konkrete Probleme in der Prognose des Todeszeitpunkts, in der Diagnose und Einschätzung von psychischen Störungen, z.B. von Depressionen und depressiven Verstimmungen, in der Bestimmung des mutmaßlichen Willens eines Patienten und schließlich in der Kontrollierbarkeit der tatsächlichen Praxis.

Für die offensichtlich nicht zu vermeidende Praxis der nichtfreiwilligen aktiven Sterbehilfe - einer Tötung ohne Kenntnis des aktuellen Wunsches eines Patienten - wird von Reform-Befürwortern auf die Möglichkeiten von Patientenverfügungen verwiesen, welche auch in extremen Situationen eine Orientierung am mutmaßlichen Willen des Leidenden ermögliche. Dagegen ist einzuwenden, dass sich der Patientenwille gerade angesichts eines Schicksalschlags auch ändern kann. Zudem ist damit zu rechnen, dass es aufgrund des ärztlichen Mitleids - wie derzeit in den Niederlanden - zu einer Praxis der nichtfreiwilligen aktiven Sterbehilfe auch bei Menschen kommt, die ihre Wünsche noch nicht oder nicht mehr äußern können.

Eine Untersuchung dieser niederländischen Entwicklungen ([23],[24],[30]) bestärkt schließlich die skeptischen Stimmen in der Debatte um eine mögliche Einführung der aktiven Sterbehilfe in unseren Krankenhäusern, Heimen und den Arztpraxen. In erster Linie ist hier die Ausweitung der Tötungspraxis auf Personenkreise zu kritisieren, die im ursprünglichen Projekt ausdrücklich davon ausgeschlossen waren: Menschen, die sich noch nicht - wie Neugeborene mit schweren Behinderungen - oder nicht mehr - wie Demente oder Menschen im chronisch vegetativen Zustand - artikulieren können, daneben auch Menschen außerhalb der Sterbephase, beispielsweise Menschen mit einer schlechten Prognose, AIDS-Kranke oder psychisch Kranke. Offensichtlich ist es im medizinischen Alltag nicht möglich, die Tötung auf Verlangen und die Tötung im Interesse des Patienten voneinander zu trennen. Selbst unter Wahrung der Sicherheitsbarrieren, die eine Ausweitung verhindern sollen, besteht in den Niederlanden ein grundsätzliches Problem darin, dass die Macht oder Entscheidungsbefugnis der Ärzte über ihre Patienten übermäßiges Gewicht erhalten hat und somit die Möglichkeit der Selbstbestimmung tendenziell eher gefährdet als gefördert wird.

\section{Die andere Tradition: Ethik des guten Lebens (und Sterbens)}

Trotz aller Angebote der Hospizbewegung, der Medizin oder der Seelsorge in der Begleitung Sterbender, bleibt die Frage nach dem Sinn des Leidens bestehen, die Frage nach der Motivation eines Menschen, eine letzte Lebensphase des Verlusts nahezu jeder Selbständigkeit überhaupt auf sich zu nehmen. Aus der Sicht des Betroffenen lautet diese Frage im Gefolge Hiobs: Warum soll ich diese Zeit der Schmerzen, der Entstellung, der Isolation, der Angewiesenheit auf andere, des Verlusts der Selbstkontrolle, warum soll ich diese sinnwidrige Zeit überhaupt auf mich nehmen?

Auf dieses „Warum“ gibt es keine befriedigende Antwort, weder eine philosophisch noch biblisch begründete. Darum bleibt lediglich die Möglichkeit, verschiedene Modelle des praktischen Umgangs mit dieser offenen Sinnfrage zu beschreiben. Ich versuche dies, indem ich das hedonistische (eigentlich als das oberflächliche zu bezeichnen), das stoische und das biblische Modell voneinander unterscheide. Während im hedonistischen Modell das Fitness-Ideal bis zum 
letzten Atemzug Gültigkeit behält (karikiert dargestellt: man will möglichst gesund sterben), wird im stoischen Modell, das auf einem starken Leib-SeeleDualismus beruht, die Schmerzunempfindlichkeit und Beharrlichkeit in den Mittelpunkt gestellt, solange noch Aussicht auf Genesung und ein selbständiges Leben besteht. In beiden Modellen bietet die Selbsttötung oder die Tötung auf Verlangen eine realistische Alternative, mit der offenen Sinnfrage umzugehen. Im biblischen Modell steht die menschliche Bezogenheit auf Gott im Zentrum, die auch im sinnlos empfundenen Leiden und über allen Tod hinaus als diese bestehen bleibt. In der Darstellung der biblischen Schriften ist der Umgang mit dem als sinnlos empfundenen Leiden einerseits von dieser Bezogenheit auf eine transzendentale Macht - der alles entspringt und die darum in ihrer ganzen Ambivalenz erlebt wird ${ }^{5}$ - andererseits aber auch von der Hoffnung auf eine Neuschöpfung geprägt. Die Selbsttötung oder eine Tötung auf Verlangen bieten in diesem Modell zumindest als vernünftige Entscheidungen - hier in Absetzung zu Akten der Verzweiflung oder der Auflehnung verstanden - keinen wirklichen Weg. Wenn schon, so wäre mit Hiob oder dem Propheten Jeremia der Tag der Geburt zu verfluchen, der alle diese Möglichkeiten erst eröffnet hat ([28], Kap. 7).

\section{Schlußbemerkung}

In einer Gesellschaft, in der verschiedene Menschenbilder und Vorstellungen von einem guten Leben und Sterben nebeneinander existieren, ist man bei der Begründung von rechtlichen Verboten in erster Linie auf die normative Diskussion angewiesen. Ein wichtiges Ergebnis dieser Untersuchung besteht in der Wahrnehmung, dass die entscheidenden Weichen in dieser normativ-ethischen Beurteilung der Sterbehilfe auf der Ebene der prinzipiellen Diskussion und in der Beurteilung der sozialen Folgen gestellt werden. Die wesentlichen Argumente, welche auf der handlungstheoretischen Ebene vorgebracht werden, stehen und fallen mit der Überzeugungskraft der prinzipiellen und folgenorientierten Überlegungen.

Im Hinblick auf die gegenwärtigen Auseinandersetzungen geht es deshalb in erster Linie darum, Verständigung über die teilweise widersprüchlichen Vorstellungen von Autonomie, Würde und Unantastbarkeit menschlichen Lebens bzw. die verschiedenen Vorstellungen von der ärztlichen Rolle und Aufgabe zu suchen. Da diese Debatten von den Welt-, Menschen- und Gottesbildern und damit von unterschiedlichen Sinneinsichten geprägt sind, erfordern sie Toleranz und die grundsätzliche Neigung, andere überhaupt verstehen zu wollen. Gleichzeitig ist es notwendig, die Ängste der Menschen angesichts möglicher zukünftiger Fehlentwicklungen und in dieser Hinsicht auch bisherige Erfahrungen in anderen Ländern ernstzunehmen. Dieses zweigleisige Vorgehen - Verständigung über die Grundlagen unseres menschlichen Zusammenlebens bei gleichzeitiger Reflexion über mögliche soziale Folgen einer veränderten Praxis - verspricht dem Ringen um humane Lösungen im Bereich der Sterbehilfe am ehesten eine solide Grundlage zu geben.

5 Vgl. Psalm 88 oder die Fluchgebete Hiobs 


\section{Literatur}

1. Aulbert E, Klaschik E, Pichlmaier H (Hrsg)(1998) Palliativmedizin - Die Alternative zur aktiven Sterbehilfe. Zur Euthanasie-Diskussion in Deutschland. Schattauer, Stuttgart New York

2. Birnbacher D (1995) Tun und Unterlassen, Stuttgart

3. Brody B (1996) Withdrawal of Treatment versus Killing of Patients. In: Beauchamp TL (Ed) Intending Death. The Ethics of Assisted Suicide and Euthanasia. Prentice Hall, Upper Saddle River, S 90-103

4. Chin AE, Hedberg K, Higginson GK, Fleming DW (1999) Legalized Physician-Assisted Suicide in Oregon. The First Year's Experience. In: NEJM 340:577-583

5. Doucet H (1998) Les promesses du crépuscule. Réflexions sur l'euthanasie et l'aide médicale au suicide. Fides Labor et Fides, Genève Saint-Laurent

6. Dworkin R (1994) Die Grenzen des Lebens. Abtreibung, Euthanasie und persönliche Freiheit. Rowohlt, Reinbek

7. Eibach U (1998) Sterbehilfe - Tötung aus Mitleid? Euthanasie und «lebensunwertes» Leben. Brockhaus-Verlag, Wuppertal (2., vollst. überarbeitete Auflage)

8. End of Life-Decisions (1997). In: Christian Bioethics 3: No. 3, Issue Editor: Eber G

9. Engelhardt Jr HT (1995) Towards a Christian Bioethics. In: Christian Bioethics 1:1-10

10. Euthanasia (1998). In: Studies in Christian Ethics 11:No. 1

11. Fischer J (1998) Handlungsfelder angewandter Ethik. Eine theologische Orientierung. Kohlhammer, Stuttgart Berlin Köln

12. Fuchs T (1997) Was heißt «töten»? Die Sinnstruktur ärztlichen Handelns bei passiver und aktiver Euthanasie. In: Ethik Med 9:78-90

13. Honecker M (1998) Euthanasie aus der Sicht der Theologie. In: Aulbert E, Klaschik E, Pichlmaier H (Hrsg) Palliativmedizin - Die Alternative zur aktiven Sterbehilfe. Zur Euthanasie-Diskussion in Deutschland. Schattauer, Stuttgart New York, S 31-39

14. Jens W, Küng H (1995) Menschenwürdig sterben. Ein Plädoyer für Selbstverantwortung. Piper, München Zürich

15. Jonsen AR (1998) The Birth of Bioethics. Oxford University Press, New York Oxford

16. Keenan JF (1999) Fallstudien, Rhetorik und die amerikanische Debatte über die ärztliche Suizidbeihilfe. In: Holderegger A (Hrsg) Das medizinisch assistierte Sterben. Zur Sterbehilfe aus medizinischer, ethischer, juristischer und theologischer Sicht. Universitätsverlag Verlag Herder, Freiburg i.Ue. Freiburg i.Br., S 157-174

17. Kissane DW, Street A, Nitschke P (1998) Seven Deaths in Darwin: Case Studies Under the Rights of the Terminally Ill Act, Northern Territory, Australia. In: Lancet 352: $1097-1102$

18. Kongregation für die Glaubenslehre (1980) Erklärung zur Euthanasie. Bonn Vatikanstadt

19. Lesch W (1997) Verletzte Würde, umstrittene Werte und gegensätzliche Interessen. Ethische Rekonstruktionen zur Debatte über die Würde der Kreatur. In: Bondolfi A, Lesch W, Pezzoli-Olgiati D (Hrsg) «Würde der Kreatur». Essays zu einem kontroversen Thema. Pano-Verlag, Zürich, S 29-46

20. Nietzsche F (1980) Die fröhliche Wissenschaft, in: Kritische Studienausgabe, Bd. 3. dtv, München (zuerst 1882)

21. 〈Playing God〉. Religious Symbols in Public Places (1995). In: Journal of Medicine and Philosophy 20: No. 4. Issue Editor: Cahill LS

22. Schöne-Seifert B (1997) Die Grenzen zwischen Töten und Sterbenlassen. In: Honnefelder L, Streffer C (Hrsg) Jahrbuch für Wissenschaft und Ethik. De Gruyter, Berlin New York, S 205-226

23. van der Maas PJ, van Delden JJM, Pijnenborg L (1992) Euthanasia and other Medical Decisions Concerning the End of Life. An Investigation Performed Upon Request of the Commission of Inquiry into Medical Practice Concerning Euthanasia. In: Health Policy 22:1-262 (Special Issue, Health Policy Monographs, Vol. 2)

24. van der Maas PJ, van der Wal G, Haverkate I, de Graaff CLM, Kester JGC, OnwuteakaPhilipsen BD, van der Heide A, Bosma JM, Willems DL (1996) Euthanasia, PhysicianAssisted Suicide, and other Medical Practices Involving the End of Life in the Netherlands, 1990-1995. In: NEJM 335:1699-1705

25. Wils J-P (1999) Sterben. Zur Ethik der Euthanasie. Schöningh, Paderborn 
26. Wise J (1998) Australian Euthanasia Law Throws Up Many Difficulties. In: BMJ 317:969

27. Wolf SM (1996) Gender, Feminism, and Death: Physician-Assisted Suicide and Euthanasia. In: Dies (Ed) Feminism and Bioethics. Beyond Reproduction. OUP, New York/Oxford, S 282-317

28. Zimmermann-Acklin M (1997) Euthanasie. Eine theologisch-ethische Untersuchung. Universitätsverlag Verlag Herder, Freiburg i.Ue. Freiburg i.Br.

29. Zimmermann-Acklin M (1998) Zwischen Suizid und Euthanasie. Erkundungen in einem Übergangsfeld. SGBE/SSEB, Genf (Folia Bioethica, Nr. 22)

30. Zimmermann-Acklin M (1999) Das niederländische Modell - Ein richtungsweisendes Konzept? In: Holderegger A (Hrsg) Das medizinisch assistierte Sterben. Zur Sterbehilfe aus medizinischer, ethischer, juristischer und theologischer Sicht, Universitätsverlag Verlag Herder, Freiburg i.Ue. Freiburg i.Br., S 351-370 